\title{
Discrete Versions of Some Classical Integrable Systems and Factorization of Matrix Polynomials
}

\author{
Jürgen Moser ${ }^{1}$ and Alexander P. Veselov ${ }^{2}$ \\ ${ }^{1}$ Forschungsinstitut für Mathematik, ETH Zürich, CH-8092 Zürich, Switzerland \\ 2 Moscow State University, Moscow, USSR
}

Received March 1, 1989; in revised form January 11, 1991

\begin{abstract}
Discrete versions of several classical integrable systems are investigated, such as a discrete analogue of the higher dimensional force-free spinning top (Euler-Arnold equations), the Heisenberg chain with classical spins and a new discrete system on the Stiefel manifold. The integrability is shown with the help of a Lax-pair representation which is found via a factorization of certain matrix polynomials. The complete description of the dynamics is given in terms of Abelian functions; the flow becomes linear on a Prym variety corresponding to a spectral curve. The approach is also applied to the billiard problem in the interior of an $\mathrm{N}$-dimensional ellipsoid.
\end{abstract}

\section{Table of Contents}

0. Introduction . . . . . . . . . . . . . . . . . . . 218

1. Discrete Version of the Dynamics of a Rigid Body . . . . . . . . . . . . . . . . 221

1.1. The Equation of "Motion" . . . . . . . . . . . . . . . . . . . . . . . . . . 221

1.2. The Solution of the Matrix Eq. (6): $\omega^{\Gamma} J-J \omega=M$. . . . . . . . . . . . . . . . 224

1.3. Isospectral Deformations . . . . . . . . . . . . . . . . . . . . . . . . . 226

1.4. The Symplectic Geometry of Eq. (6) . . . . . . . . . . . . . . . . . . . . 227

1.5. The Integration of the Discrete Euler Equation . . . . . . . . . . . . . . . 230

1.6. Explicit Formulas for the Discrete Dynamics of the 3-Dimensional Rigid Body. . 233

2. The Discrete Dynamics on Stiefel Manifolds and the Heisenberg Chain

with Classical Spins . . . . . . . . . . . . . . . . . . . . . . 235

2.1. The Equation of the Dynamics and Isospectral Deformations . . . . . . . . . 235

2.2. Discrete Version of the C. Neumann System and the Heisenberg Chain with Classical Spins . . . . . . . . . . . . . . . . . . . . 236

3. The Billiard Inside an Ellipsoid . . . . . . . . . . . . . . . . . . . . . . . . . . . . . . 238

3.1. The Splittings and Isospectral Deformations. . . . . . . . . . . . . . . . . . . . . . . . . 239

3.2. Connection Between the Ellipsoidal Billiard and the Discrete C. Neumann System . . . . . . . . . . . . . . . . . . . . . . . . . . . 241

References . . . . . . . . . . . . . . . . . 242 


\section{Introduction}

In this paper we study a class of discrete integrable systems which are closely related to problems occurring in mathematical physics such as the Heisenberg model for classical spins or the billiard problem in the interior of an ellipsoid. A discrete system can be viewed as the iterates of a symplectic mapping, the time $t \in \mathbb{Z}$ being the number of iterations. Such a system will be called integrable if it possesses sufficiently many integrals which are in involution with respect to a symplectic structure.

To describe such a discrete system we take as starting point a variational principle

$$
\delta S=0
$$

for a functional $S=S(X)$ defined on the space of sequences $X=\left(X_{k}\right), k \in \mathbb{Z}$ by a formal sum

$$
S=\sum_{k \in \mathbb{Z}} \mathscr{L}\left(X_{k}, X_{k+1}\right) .
$$

Here $X_{k}$ are points on a manifold $\mathscr{M}^{n}$ and $\mathscr{L}$ is a function on $Q^{2 n}=\mathscr{M}^{n} \times \mathscr{M}^{n}$. The Euler-Lagrange equation of such a functional are second order difference equations (see Sect. 1) and $k \in \mathbb{Z}$ plays the role of the discrete time.

This description is to be viewed as the discrete analogue of the Hamilton principle $\delta S=0$ for

$$
S=\int \mathscr{L}(q, \dot{q}) d t,
$$

and the related symplectic flow can, as usual, be defined via the Legendre transform provided $\operatorname{det}\left(\mathscr{L}_{\dot{q} \dot{q}}\right) \neq 0$. Similarly, one can define a symplectic structure on $Q^{2 n}$ under an appropriate nondegeneracy assumption (see Sect. 1, Sect. 2, (8) and [1]). We will call such a system "integrable" if there are sufficiently many integrals which are in involution with respect to this symplectic structure.

As an example of this setup we mention the Heisenberg chain with classical spins, where

$$
M=S^{2}=\left\{x \in \mathbb{R}^{3},|x|=1\right\}
$$

and

$$
\mathscr{L}=(x, J y),
$$

where $J$ is a symmetric matrix, which we may take to be diagonal.

The corresponding chain of quantum spins $\frac{1}{2}$ (so-called $X Y Z$ Heisenberg model) was investigated by Faddeev and Takhtajan [2] in the framework of the quantum inverse scattering method, using the fundamental results by Baxter [3]. As it was shown by Pokrovsky and Khokhlachev [4], the problem of finding some special eigenfunctions in the quantum $X Y Z$-model leads to the stationary equation $\delta S=0$ for the Heisenberg chain with classical spins. For this discrete system Granovsky and Zhedanov $[5,6]$ found two algebraic integrals and special solutions. The integrability of these systems, even for arbitrary dimension $n$ of the sphere: $M=S^{n}$, was shown by one of the authors ([7], see also [1]), where the general solution was described in terms of $\theta$-functions, generalizing the connection between the spectral theory of one-dimensional Schrödinger operators and the classical Neumann systems derived in $[8,9]$ ). 
The main problem to be discussed in the first part of this paper is a chain of orthogonal matrices: We take $\mathscr{M}^{n}=O(N), n=\frac{N(N-1)}{2}$, and

$$
\mathscr{L}(X, Y)=\operatorname{tr}\left(X J Y^{T}\right),
$$

where $J^{1}$ is a positive symmetric matrix. This problem was introduced in [1] where it was shown that in the continuous limit this problem leads to the Euler problem for force-free motion of a rigid body as it was generalized by Arnold to arbitrary dimensions.

Alternately, one could use the positive Lagrangian $\frac{1}{2} \operatorname{tr}\left((X-Y) J(X-Y)^{T}\right)$. Indeed, for $X, Y \in \mathcal{O}(N)$ this expression agrees with $\operatorname{tr} J-\operatorname{tr}\left(X J Y^{T}\right)$, i.e. differs from $-\mathscr{L}(X, Y)$ only by a constant.

For the cases $N=3, N=4$ this system was shown to be integrable by explicit construction of commuting integrals [1]. In Sect. 3 we will establish the integrability of this system for all $N$ by using the discrete version of the isospectral technique, which leads to the complete description of the dynamics of this system in terms of Abelian functions. The flow is quasi-periodic in the discrete time parameter $k$ and is linear on a Prym variety.

We indicate the approach underlying the solution of this problem. It is based on the construction of an isospectral mapping on a class of matrices $(L)$ into itself, analogous to the Lax approach in the continuous case in which the differential equation is cast in the form $\dot{L}=[L, A]$ for some class of linear operators $(L)$.

Finding the class of matrices $(L)$ and the isospectral deformation is a hit-ormiss game and depends on good guesses. In the discrete case it turns out to be connected with a factorization of matrices. We recall the beautiful observation by Symes [11] that the QR-algorithm of Jacobi matrices is closely linked to the Toda flow: The QR-algorithm, an important device in numerical analysis for diagonalization of matrices, consists in factoring a real nondegenerate matrix $L$ into a product $L=Q R$ of an orthogonal matrix $Q$ and an upper triangle matrix $R$ with positive diagonal elements. Now the mapping

$$
L=Q R \rightarrow L_{1}=R Q=Q_{1} R_{1}
$$

gives rise to an isospectral map since $L_{1}=Q^{-1} L Q$. It was Symes' observation [11] that the application of this process to $L=\exp K$, where $K$ is a symmetric tridiagonal (or Jacobi-) matrix leads to an integrable mapping which is interpolated by the Toda flow. This idea has been extended to more general classes of matrices by Deift et al., see [12].

Although this mapping has no variational description, this idea turns out to be fruitful also for the problems at hand. The main new feature is that we start with a class of certain quadratic matrix polynomials

$$
L(\lambda)=A_{0}+A_{1} \lambda+A_{2} \lambda^{2}
$$

and a suitable factorization

$$
L(\lambda)=\left(B_{0}+B_{1} \lambda\right)\left(C_{0}+C_{1} \lambda\right)=B(\lambda) C(\lambda) .
$$

\footnotetext{
1 We denote the "moment of inertia" by $J$ and not, as is customary, by $I$, to avoid confusion with the identity matrix
} 
If such a factorization exists and can be defined in a unique way then it gives rise to an isospectral mapping $L(\lambda) \rightarrow L_{1}(\lambda)$ by exchanging the factors:

$$
L_{1}(\lambda)=C(\lambda) B(\lambda)=B_{1}(\lambda) C_{1}(\lambda)=B^{-1}(\lambda) L(\lambda) B(\lambda) .
$$

This can be viewed as a discrete analogue of the Lax representation.

The main difficulty is, of course, to find the class of matrix polynomials together with a factorization in such a way that it corresponds to the dynamics of the given problem. Moreover, even if one has such a factorization it is generally not unique and the above procedure gives rise only to a correspondence, i.e. a multiple valued mapping; this is in good agreement with the fact that frequently the difference equation $\delta S=0$ gives rise to such correspondences.

In the problem of orthogonal chains we are able to describe such a class of matrix polynomials and a corresponding factorization which can be made unique by specifying a suitable splitting of the spectrum. From this Lax representation we will find the integrals as well as the algebraic curve on whose Jacobian variety the flow becomes linear in $k$. A general theory of factorization of matrix polynomials can be found in [13]. The special factorization derived here (see Sect. 1) uses standard ideas from [13]; it involves orthogonal matrices and may be of interest in itself. Using the ideas of "finite-gap" integration [14] in the matrix case developed by Dubrovin [15] we exhibit explicit formulas for the dynamics in the case $n=3$ in terms of classical elliptic functions.

In Sect. 2 we discuss some generalizations of this system, including the above mentioned Heisenberg model with classical spins. We take $\mathscr{M}$ as the manifold of rectangular $n \times N$ matrices $X, n \leqq N$ for which $X X^{T}=I_{n}$ and define the Lagrange function by

$$
\mathscr{L}(X, Y)=\operatorname{tr}\left(X J Y^{T}\right),
$$

where $J$ is a symmetric $N \times N$ matrix. In other words $\mathscr{M}$ is the Stiefel manifold $V_{n, N}$ of orthonormal $n$-frames in $\mathbb{R}^{N}$. For $n=1, N=3$ this represents the Heisenberg model and for $n=N$ we obtain the chain of orthogonal matrices described above. For $n=1$ we show how the factorization procedure leads to the hyperelliptic curves and formulas involving $\theta$-functions as in [1]. In the general case $1<n<N$ we exhibit the corresponding factorization problem without full treatment.

The last section (Sect. 3) is devoted to the billiard problem in the interior of an ellipsoid in $\mathbb{R}^{N}$. This problem also fits into the above framework. The relevant class of matrices for the Lax representation agree with those introduced in [10] for the study of the geodesic flow on an ellipsoid - probably the oldest nontrivial integrable system in arbitrary dimensions. This class of matrices fits into the procedure of Sect. 1. Finally, we will establish a connection between this billiard problem and a discrete version of the Neumann problem, where a certain symmetry of this system, found in [1], will play a crucial role. In the continuous case such a connection between the geodesic flow on the ellipsoid and the Neumann system was discovered by Knörrer with the help of the Gauss mapping of the ellipsoid [16]. However, the connection described here is of a different nature.

In the above discussion we referred frequently to a discrete version of a continuous system, as in the case of the billiard problem inside an ellipsoid and the geodesic flow on the same ellipsoid - whose orbits are obtained as limits of tangential billiard shots. Another example is the above mentioned chain of 
orthogonal matrices and the corresponding continuous system of the force free top. Without trying to be precise we require in all these cases a) that the discrete system tends to the continuous system under a limit process and b) that both systems are integrable and are given by "natural" variational problems.

As a rule it is easy to go from a discrete system to a continuous one without destroying integrability. However, the converse is much more difficult, as is often the case if one wants to preserve some structure under discretization of a continuous system. Of course, one could take the "time $\varepsilon$ " mapping of a flow but that is usually not described by a simple variational problem. The difficulty is to preserve the integrability under discretization. In this sense our method may be of interest since it provides an approach to the construction of an integrable discretization for the continuous system with known Lax representation, polynomially depending on an additional "spectral" parameter $\lambda$. The importance of representations of this type became clear after the paper of Novikov [17]. They exist for most of the known integrable hamiltonian systems and are related to the theory of Lie algebras (see $[18,19])$. From this point of view the nature of the factorization procedure calls for a better understanding. Notice that in all our examples the matrix polynomials $L(\lambda)$ have the property $L^{*}(\lambda)=L(\lambda)$, where $L^{*}(\lambda)$ $=L^{T}(-\lambda)$, and the corresponding factorizations $L(\lambda)=B(\lambda) C(\lambda)$ satisfy the condition $B(\lambda)=C^{*}(\lambda)$.

In a forthcoming paper [29] it is shown that the factorization of certain linear(!) matrix polynomials, introduced in [10], leads to the billiard problems in domains on the sphere and the Lobachevsky space, bounded by conic sections.

The present paper was completed in February 1989 and has been circulated as a preprint of the Forschungsinstitut für Mathematik Zürich. For various reasons its publication has been delayed. We added some relevant new references at the end of this revised paper. In particular, we draw attention to the interesting work by Deift, $\mathrm{Li}$, and Tomei [32] in which the systems considered in the present paper are related to loop groups. Moreover, it is shown how the discrete mappings considered here can be interpolated by integrable Hamiltonian flows.

\section{The Discrete Version of the Dynamics of a Rigid Body}

\subsection{The Equations of "Motion"}

We consider the functional $S(X)$, determined by a formal sum

$$
S=\sum_{k} \operatorname{tr}\left(X_{k} J X_{k+1}^{T}\right)
$$

on the sequences $X=\left(X_{k}\right)$ with $X_{k} \in O(N)$, i.e. orthogonal $N$ by $N$ matrices. The stationary points of $S$ are described by the equation $\delta S=0$ or

$$
X_{k+1} J+X_{k-1} J=\Lambda_{k} X_{k},
$$

where $\Lambda_{k}=\Lambda_{k}^{T}$ is a matrix Lagrange multiplier, which is determined in such a way that $X_{k} X_{k}^{T}=I$. $\Lambda_{k}$ is uniquely determined by $X_{k-1}, X_{k}, X_{k+1}$, but as we will see later, not uniquely by $X_{k-1}, X_{k}$; it is a complicated function of $X_{k-1}, X_{k}$.

Therefore we use the Euler description of the dynamics. This can be done in the following way. Rewrite (2) as

$$
X_{k+1} J X_{k}^{T}+X_{k-1} J X_{k}^{T}=\Lambda_{k}=\Lambda_{k}^{T}=X_{k} J X_{k+1}^{T}+X_{k} J X_{k-1}^{T} .
$$


Introducing $m_{k}=X_{k} J X_{k-1}^{T}-X_{k-1} J X_{k}^{T}$ we see that the last Eq. (3) means that $m_{k+1}=m_{k}$. The conservation of $m_{k}$, which is the discrete analogue of the angular momentum in space [20] is the consequence of the left-invariance of $\mathscr{L}(X, Y)$ (see [1]). In the variables fixed relative to the body we have the "angular velocity" $\omega_{k}=X_{k}^{T} X_{k-1}=X_{k}^{-1} X_{k-1} \in O(N)$ and "angular momentum with respect to the body" $M_{k}=X_{k-1}^{-1} m_{k} X_{k-1}=\omega_{k}^{T} J-J \omega_{k} \in O(N)^{*}$ and thus Eq. (3) can be rewritten as a "discrete Euler-Arnold equation" [1]

$$
\left\{\begin{array}{l}
M_{k+1}=\omega_{k} M_{k} \omega_{k}^{-1}, \\
M_{k}=\omega_{k}^{T} J-J \omega_{k}, \quad \omega_{k} \in O(N)
\end{array}\right.
$$

In the continuous limit when $X_{k}=X\left(t_{k}\right), t_{k}=t_{0}+k \varepsilon, \omega_{k}=X_{k}^{-1} X_{k-1} \approx I-\varepsilon \Omega\left(t_{k}\right)$, $\omega_{k}=X_{k}^{-1} X_{k-1}$ and $M_{k}=\omega_{k}^{T} J-J \omega_{k} \approx \varepsilon(J \Omega+\Omega J)=\varepsilon M\left(t k_{k}\right), M=J \Omega+\Omega J$, this Eq. (4) becomes the usual Euler-Arnold equations for the motion of the $N$-dimensional rigid body

$$
\left\{\begin{array}{l}
\dot{M}=[M, \Omega], \\
M=J \Omega+\Omega J, \quad \Omega \in o(N) .
\end{array}\right.
$$

The main new feature of the discrete system (4) is the connection between $M$ and $\omega$ :

$$
M=\omega^{T} J-J \omega, \quad \omega \in O(N), \quad M^{T}=-M,
$$

which we need to solve to find $\omega$. In fact such $\omega$ is not unique (see below) and $\omega_{k+1}$ is not uniquely determined by (4), which therefore leads to a correspondence and not to a mapping.

We discuss the symplectic properties of this correspondence (see also [1]). The Eq. (2) is a particular case of the Lagrangian equations $\delta S=0$ for the functional

$$
S=\sum_{k \in \mathbb{Z}} \mathscr{L}\left(X_{k}, X_{k+1}\right), \quad X_{k} \in \mathscr{M}^{n}, \quad \mathscr{L}=\mathscr{L}(x, y)
$$

(see the Introduction), which in an appropriate coordinate system $(x, y)$ on $Q^{2 n}$ $=\mathscr{M}^{n} \times \mathscr{M}^{n}$ can be written as

$$
\delta S=0, \quad \frac{\partial \mathscr{L}}{\partial x}\left(X_{k}, X_{k+1}\right)+\frac{\partial \mathscr{L}}{\partial y}\left(X_{k-1}, X_{k}\right)=0 .
$$

The submanifold $\Gamma^{2 n}$ in $Q^{2 n} \times Q^{2 n}$, defined by

$$
x^{\prime}=y, \quad \frac{\partial \mathscr{L}}{\partial x}\left(x^{\prime}, y^{\prime}\right)+\frac{\partial \mathscr{L}}{\partial y}(x, y)=0
$$

determines generally some correspondence between subsets of $Q^{2 n}$.

On $Q^{2 n}$ one can define a closed 2-form $\sigma$ by

$$
\sigma=\frac{\partial^{2} \mathscr{L}}{\partial x \partial y} d x \wedge d y
$$

or

$$
\sigma=d \beta=d\left(\frac{\partial \mathscr{L}}{\partial x}(x, y) d x\right), \quad \beta=\frac{\partial \mathscr{L}}{\partial x} d x
$$

where $d \mathscr{L}=\frac{\partial \mathscr{L}}{\partial x} d x+\frac{\partial \mathscr{L}}{\partial y} d y$ is a natural decomposition of the 1 -form on $Q^{2 n}$. 
The submanifold $\Gamma^{2 n}$ is isotropic for the form $\sigma^{\prime}-\sigma$ on $Q^{2 n} \times Q^{2 n}$. Indeed

$$
\begin{aligned}
\beta^{\prime}-\left.\beta\right|_{\Gamma^{2 n}} & =\left.\frac{\partial \mathscr{L}}{\partial x}\left(x^{\prime}, y^{\prime}\right) d x^{\prime}\right|_{\Gamma^{2 n}}-\left.\frac{\partial \mathscr{L}}{\partial x}(x, y) d x\right|_{\Gamma^{2 n}} \\
& =-\left.\frac{\partial \mathscr{L}}{\partial y}(x, y) d y\right|_{\Gamma^{2 n}}-\left.\frac{\partial \mathscr{L}}{\partial x}(x, y) d x\right|_{\Gamma^{2 n}}=-\left.d \mathscr{L}\left(x, x^{\prime}\right)\right|_{\Gamma^{2 n}} .
\end{aligned}
$$

We see that $\mathscr{L}$ is the generating function of the mapping, determined locally by $(8)$ in the domain of nondegeneracy of $\sigma$ :

$$
\operatorname{det}\left|\frac{\partial^{2} \mathscr{L}}{\partial x \partial y}\right| \neq 0
$$

and therefore this mapping is symplectic with respect to the symplectic structure $\sigma$.

In this connection it is useful to introduce the discrete version of the Legendre transformation $\tau$ of $Q^{2 n}$ into $T^{*} \mathscr{M}$. It is defined by

$$
\tau:(x, y) \rightarrow(x, p), \quad p d x=\mathscr{L}_{x}(x, y) d x,
$$

where $p$ is the fiber coordinate and $\alpha=p d x$ the standard 1-form on $T^{*} \mathscr{M}$. Thus the pullback of this form is $\tau^{*} \alpha=\beta=\mathscr{L}_{x} d x$, and the standard symplectic form $d \alpha$ on $T^{*} \mathscr{M}$ pulls back to $\tau^{*} d \alpha=d \beta=\sigma$, which is nondegenerate whenever $\tau$ is noncritical. Generally, $\tau$ has only a local inverse.

We discuss the concepts in our case where $\mathscr{M}=O(N), \mathscr{L}(X, Y)=\operatorname{tr}\left(X J Y^{T}\right)$ and

$$
\beta=\operatorname{tr}\left(d X J Y^{T}\right) .
$$

To describe the Legendre transformation $\tau: O(N) \times O(N) \rightarrow T^{*} O(N)$ we identify $T^{*} O(N)$ and $T O(N)$ via the bilinear form $\operatorname{tr}\left(A B^{T}\right)$ so that

$$
\tau:(X, Y) \rightarrow(X, P), \quad P=Y J-X S \in T_{X} O(N),
$$

where $S=S^{T}$ is so chosen that $X^{T} P$ is skew symmetric, i.e.

$$
P=\frac{1}{2}\left(Y J-X J Y^{T} X\right) \text {. }
$$

The standard 1-form $\alpha=\operatorname{tr}\left(P^{T} d X\right)$ is taken into $\beta=\operatorname{tr}\left(d X J Y^{T}\right)$, since $X^{T} d X$ is skew symmetric. The standard sympletic form $d \alpha$ on $T^{*} O(N)$ is mapped into

$$
\sigma=d \beta=\operatorname{tr}\left(d X J \wedge d Y^{T}\right) .
$$

We record that this 2 -form $\sigma$ is the pullback of the standard symplectic form on $T^{*} O(N)$ under $\tau$; it is nondegenerate at all noncritical points of $\tau$.

If we define locally a mapping $\phi:(X, Y) \rightarrow\left(X^{\prime}, Y^{\prime}\right)$ by selecting a branch of the correspondence

$$
Y^{\prime} J+X J=\Lambda X^{\prime}, \quad X^{\prime}=Y ; \quad \Lambda^{T}=\Lambda,
$$

where $X, Y, X^{\prime}, Y^{\prime} \in O(N)$, then this mapping preserves $\sigma$. Equivalently, the mapping $\tau \phi \tau^{-1}$, locally defined near regular values of $\tau$, preserves the standard symplectic structure as well as the corresponding Poisson structure on $T^{*} O(N)$.

Since both $\tau, \psi$ commute with left translation of $O(N)$ we can reduce the map $\tau \phi \tau^{-1}$ to a mapping of $o^{*}(N)$ by projecting $(X, P) \rightarrow X^{T} P \in o(N)$. The resulting reduced mapping $\psi: o^{*}(N) \rightarrow o^{*}(N)$ is the one defined by (4) taking $M=M_{k}$ into 
$M^{\prime}=M_{k+1}$, i.e.

$$
\psi: M=\omega^{T} J-J \omega \rightarrow M^{\prime}=J \omega^{T}-\omega J .
$$

Here it is crucial to solve the matrix equation $M=\omega^{T} J-J \omega$ for $\omega \in O(N)$, a question which will be discussed completely in Subsect. 1.2.

Now it is well known that the reduction of $T^{*} O(N)$ to $o^{*}(N)$ takes the standard Poisson structure of $T^{*} O(N)$ (up to a constant nonzero factor) into the Lie-Poisson structure

$$
\{f, g\}=\operatorname{tr}\left(M\left[f_{M}, g_{M}\right]\right), \quad f, g \in C^{\infty}(o(N))
$$

on $o^{*}(N)$ which we identify again with $o(N)$; here $f_{M}$ denotes the skew-symmetric matrix of partial derivatives $\partial f / \partial M_{i j}$. This proves that the mapping $\psi: M \rightarrow M^{\prime}$ of (4) preserves this Poisson structure, which agrees with the Poisson structure preserved by the usual continuous rigid body motion given by (5).

This reduction is the discrete version of the well known reduction procedure [20] for Hamiltonian systems. For the derivation of (11) see also [30, 31].

Our next goal is to show that this mapping is integrable, i.e. preserves sufficiently many functions $F_{i}$, which are in involution with respect to the above Poisson structure. As a matter of fact it turns out that these "integrals" have the same form as in the continuous case which are known to be in involution.

\subsection{The Solution of the Matrix Eq. (6): $\omega^{T} J-J \omega=M$}

We have to solve two crucial problems: a) to define the mapping $\phi$ in a unique way by selecting a branch of the correspondence and b) to verify that this mapping is integrable. Both these problems can be reduced to an appropriate factorization problem for a matrix polynomial, as we will show now.

The first problem, to construct a well defined map $\phi:(X, Y) \rightarrow\left(X^{\prime}, Y^{\prime}\right)$ whose graph belongs to the correspondence (9) reduces to finding a well defined solution $\omega \in O(N)$ of the matrix equation:

$$
M^{\prime}=\left(\omega^{\prime}\right)^{T} J-J \omega^{\prime}
$$

for a given skew-symmetric matrix $M$. Indeed, setting $\omega=Y^{T} X, M=\omega^{T} J-J \omega$, $M^{\prime}=\omega M \omega^{-1}=J \omega^{T}-\omega J$, then $X^{\prime}, Y^{\prime}$ of $(9)$ is given by $X^{\prime}=Y, Y^{\prime}=X^{\prime}\left(\omega^{\prime}\right)^{T}$.

This problem is, in fact, equivalent to finding a definite inverse for the Legendre transformation $\tau:(X, Y) \rightarrow(X, P)$, since

$$
X^{T} P=\frac{1}{2}\left(X^{T} Y J-J Y^{T} X\right)=\frac{1}{2}\left(\omega^{T} J-J \omega\right) .
$$

Hence a solution $\omega$ of this equation gives rise to $Y=X \omega^{T}$, thus defining $\tau^{-1}$.

The crucial observation is contained in the following

Lemma. The matrix Eq. (6) is equivalent to the factorization

$$
\left(I-\lambda M-\lambda^{2} J^{2}\right)=\left(\omega^{T}+\lambda J\right)(\omega-\lambda J) .
$$

The proof is an obvious verification, which shows also that the solution $\omega$ is necessarily an orthogonal matrix. It turns out that the choice of the solution $\omega$ is fixed by the corresponding factorization of the determinant

$$
P(\lambda)=\operatorname{det}\left(I-\lambda M-\lambda^{2} J^{2}\right)=p(-\lambda) p(\lambda) .
$$


We will prove below

Theorem 1. Assume that for the real skew symmetric matrix $M$ the polynomial $P(\lambda)$ $=P(-\lambda)$ admits a splitting

$$
P(\lambda)=p(\lambda) p(-\lambda) ;
$$

with a real polynomial $p(\lambda)$ satisfying

$$
|p(\lambda)|+|p(-\lambda)|>0 \quad \text { for all } \quad \lambda \in \mathbb{C}
$$

then there exists a unique matrix $\omega \in O(N)$ satisfying (12) and

$$
\pm p(\lambda)=\operatorname{det}(\omega-\lambda J) \text {. }
$$

We postpone the proof of this theorem to Subsect. 1.4. We discuss the splitting of the determined $P(\lambda)$. Since $M+M^{T}=0$ one has $P(\lambda)=P(-\lambda)$ and the set $\Sigma$ of all roots of $P(\lambda)$ satisfies $\Sigma=-\Sigma$. The factorization (13) corresponds to a splitting $\Sigma=\Sigma_{+} \cup \Sigma_{-}$into disjoint sets $\Sigma_{+}, \Sigma_{-}$satisfying

$$
\bar{\Sigma}_{+}=\Sigma_{+}, \quad \bar{\Sigma}_{-}=\Sigma_{-}, \quad \Sigma_{+}=-\Sigma_{-},
$$

where $\Sigma_{+}$is the zero set of the real polynomial $p(\lambda)$. Here we denote for any set $A$ $\subset \mathbb{C}$ by $\bar{A}$ the set of all $\bar{a}, a \in A$ and by $-A$ the set of $(-a), a \in A$. Any such splitting gives rise to such a factorization (12) and thus to a solution of (6). Obviously the possibility of such a factorization requires that $P(\lambda)$ has no roots on the imaginary axis. In this case one factorization is obtained by taking for $\Sigma_{+}$the roots of $P(\lambda)$ in the right half plane, and $\Sigma_{-}=-\Sigma_{+}$.

We give an outline of the proof of Theorem 1 under the assumption that the roots of $P(\lambda)$ are distinct, leaving the complete proof of the general case for later. Denote the elements of $\Sigma_{+}$by $\lambda_{1}, \lambda_{2}, \ldots, \lambda_{N}$ (and $\Sigma_{-}=\left\{-\lambda_{1},-\lambda_{2}, \ldots,-\lambda_{N}\right\}$ ). Then there exist eigenvectors $\psi_{k}:\left(I-\lambda_{k} M-\lambda_{k}^{2} J^{2}\right) \psi_{k}=0$. Because of the nondegeneracy of $\omega^{T}+\lambda_{i} J$ we have from the factorization (12)

$$
\left(\omega-\lambda_{k} J\right) \psi_{k}=0,
$$

or, equivalently

$$
\omega \psi=J \psi \Lambda,
$$

where $\psi$ is the $N$ by $N$ matrix with columns $\psi_{k}$ and $\Lambda=\operatorname{diag}\left(\lambda_{1}, \lambda_{2}, \ldots, \lambda_{N}\right)$.

If $\psi$ is invertible then

$$
\omega=J \psi \Lambda \psi^{-1}
$$

defines the desired solution. It can be shown that $\psi$ is indeed nondegenerate and that (14) actually defines the solution of (6) which, moreover, is real and orthogonal. But in Subsect. 1.4 we present another approach to the solution of (6) which is a bit more general. This proof will also provide the nondegeneracy of $\psi$ and complete the above considerations. In this connection the concepts of symplectic geometry will turn out to be useful.

We note that the solutions $\omega \in O(N)$ so obtained have the property that the polynomials $p(\lambda)= \pm \operatorname{det}(\omega-\lambda J)$ and $p(-\lambda)$ have no common roots. In other words, any two eigenvalues $\lambda, \lambda^{\prime}$ of $\omega J^{-1}$ satisfy $\lambda+\lambda^{\prime} \neq 0$. We will denote the set of these matrices by $E$, i.e.

$$
E=\{\omega \in O(N),|p(\lambda)|+|p(-\lambda)|>0 \forall \lambda \in \mathbb{C} ; p(\lambda)=\operatorname{det}(\omega-\lambda J])\} .
$$


This is clearly an open subset of $O(N)$ containing a neighborhood of the identity. Since $p(\lambda)$ does not vanish on the imaginary axis $E$ decomposes into several components, depending on how many roots of $p(\lambda)$ lie in the left half plane.

With the aid of Theorem 1 it is easy to define a mapping $\phi$ in a unique way. We do this in the reduced form and rewrite the above factorization (12) in the form

$$
\left(I-\lambda M-\lambda^{2} J^{2}\right)=A^{T}(-\lambda) A(\lambda) ; \quad A(\lambda)=\omega-\lambda J .
$$

Then the image point $M^{\prime}=\psi M$ is given by

$$
\left(I-\lambda M^{\prime}-\lambda^{2} J^{2}\right)=A(\lambda) A^{T}(-\lambda),
$$

where the two factors were exchanged. This equation is readily verified from (10). Thus the determinants $P(\lambda), P^{\prime}(\lambda)$ of (16), (17) respectively are identical. By Theorem 1 any splitting $P(\lambda)=p(\lambda) p(-\lambda)$ gives rise to a unique factorization. Hence for (17) there exists a unique $\omega^{\prime}$ and $A^{\prime}(\lambda)=\omega^{\prime}-\lambda J$ with

$$
\left(I-\lambda M^{\prime}-\lambda^{2} J^{2}\right)=A^{\prime T}(-\lambda) A^{\prime}(\lambda)
$$

with

$$
\operatorname{det}\left(\omega^{\prime}-\lambda J\right)=\operatorname{det}(\omega-\lambda J)=p(\lambda)
$$

This gives rise to a well defined mapping $\omega \rightarrow \omega^{\prime}$ taking $E$ into itself. The uniqueness is achieved by the requirement (18) which is consistent with iterations of the mapping. ${ }^{2}$

Similarly, the mapping $\phi:(X, Y) \rightarrow\left(X^{\prime}, Y^{\prime}\right)$ is well defined on the left invariant set

$$
\widetilde{Q}=\left\{X, Y \in O(N), Y^{T} X \in E\right\}
$$

and given by $\left(X^{\prime}, Y^{\prime}\right)=\left(Y, Y\left(\omega^{\prime}\right)^{T}\right)$ if $\omega=Y^{T} X$. We mention that one easily verifies that $\widetilde{Q}$ is precisely the set of regular points of the Legendre transform $\tau$. Thus $\sigma$ is nondegenerate on $\widetilde{Q}$ making $\widetilde{Q}$ a symplectic manifold.

\subsection{Isospectral Deformations}

From the above considerations we obtain the desired integrals. For this purpose we write the mapping in terms of an isospectral deformation. The Eq. (4) is already in this form but yields only $k=\left[\frac{N}{2}\right]$ "trivial" integrals $\operatorname{tr}\left(M^{2 v}\right), v=1,2, \ldots, k$, (in fact, these are coadjoint invariants of $O(N)$ ) which is not sufficient for complete integrability. As was pointed out by Novikov [17] it is crucial to have such a representation for a matrix depending polynomially on a parameter $\lambda$. form

In our case we make use of (16), (17) to obtain for the mapping $\psi: M \rightarrow M^{\prime}$ the

$$
\left(I-\lambda M^{\prime}-\lambda^{2} J^{2}\right)=A(\lambda)\left(I-\lambda M-\lambda^{2} J^{2}\right) A^{-1}(\lambda)
$$

\footnotetext{
2 We note that this mapping $\omega \rightarrow \omega^{\prime}$ in $E$ is the product of two involutions: We observe that to any $\omega \in E \subset O(N)$ we can, by Theorem 1, associate a second solution $\omega_{*}$ of $(6)$ for which $\operatorname{spec}\left(\omega_{*} J^{-1}\right)$ $=-\operatorname{spec}\left(\omega J^{-1}\right)$, i.e. for which $p_{*}(\lambda)=\operatorname{det}\left(\omega_{*}-\lambda J\right)=(-1)^{n} p(-\lambda)$. The map $j_{1}: \omega \rightarrow \omega_{*}$ is clearly an involution on $E$. To bring the spectrum back we use the trivial involution $j_{2}: \omega \rightarrow-\omega^{T}$. One verifies readily that our mapping is given by $j_{1} \circ j_{2}$, i.e. $\omega^{\prime}=j_{1} j_{2} \omega$
} 
or equivalently

$$
L^{\prime}(\lambda)=M^{\prime}+\lambda J^{2}=A(\lambda)\left(M+\lambda J^{2}\right) A^{-1}(\lambda) .
$$

Consequently the polynomials $f_{k}(M, \lambda)=\operatorname{tr}\left(M+\lambda J^{2}\right)^{k}$ are integrals of $\psi$. In other words, the characteristic polynomial $\operatorname{det}(L(\lambda)-\mu I)$ is preserved by $\psi$, or in homogeneous form

$$
\operatorname{det}\left(v M+\lambda J^{2}-\mu I\right)=\sum_{2 \alpha+\beta+\gamma=N} v^{2 \alpha} \lambda^{\beta} \mu^{\gamma} Q_{\alpha \beta \gamma}(M),
$$

the coefficients $Q_{\alpha \beta \gamma}(M)$ for $\alpha \geqq 1,2 \alpha+\beta+\gamma=N$ provide $k^{2}$ integrals if $N=2 k$, or $k(k+1)$ integrals if $N=2 k+1$.

These integrals $f_{k}(M, \lambda)$ or $Q_{\alpha \beta \gamma}(M)$ are precisely the same as for the EulerArnold Eq. (5). Indeed, for these equations the Lax representation was found by Manakov [22] in the form

$$
\frac{d}{d t}\left(M+\lambda J^{2}\right)=\left[M+\lambda J^{2}, \Omega+\lambda J\right]
$$

showing that also $f_{k}(M, \lambda)$, or $Q_{\alpha \beta \gamma}(M)$ are integrals of the motion.

It is well known that these functions are in involution with respect to the Poisson structure (11) and independent, making the system (5) completely integrable. Since our discrete map $\psi: M \rightarrow M^{\prime}$ preserves the same Poisson structure (11) as well as the functions $f_{k}(M, \lambda)$ we conclude that $\psi$ is also integrable. We summarize these results in

Theorem 2. The discrete Euler Eq. (4) is equivalent to the isospectral deformation

$$
L_{k+1}=A_{k} L_{k} A_{k}^{-1}, \quad \operatorname{det} A_{k+1}=\operatorname{det} A_{k},
$$

where $L_{k}=M_{k}+\lambda J^{2}, A_{k}=\omega_{k}-\lambda J, M_{k+1}=\psi\left(M_{k}\right)$. This mapping $\psi$ preserves the Poisson structure (11) and is completely integrable. It preserves the same Poisson structure and integrals $F_{i}$ as the continuous system (5) for the motion of the rigid body.

The "integration" of this system is now rather straightforward, since the integration of the continuous case is known: The nonsingular compact level sets $T_{c}=\bigcap_{i}\left(F_{i}=c_{i}\right)$ consist of a finite union of tori, according to well known arguments [20]. Since our mapping $\psi$ preserves the Poisson structure (11) as well as the functions $F_{i}$ it commutes with all commuting Hamiltonian flows generated by the $F_{i}$, defined by $\dot{M}=\left[M, \nabla F_{i}\right]$. On each such torus our mapping $\psi$ must be a translation with respect to the affine structure, determined by these flows. In this case this mapping can be represented as a shift along the trajectory of a certain integral $H$ (see [1] and Subsect. 1.5).

We will show that in our case $T_{c}$ is the real part of a complex Abelian variety of the curve $\operatorname{det}\left(M+\lambda J^{2}-\mu I\right)=0$, and Eq. (4) determines a translation on it. In fact, it turns out to be the same Prym variety occurring in the integration of the EulerArnold equation (see, for example, [21] and [33]).

\subsection{The Symplectic Geometry of Eq. (6)}

First of all we write (6) as

$$
\omega^{-1} J-J \omega=M, \quad \omega \omega^{T}=I,
$$


and introducing $W=\omega^{-1} J$ we obtain the quadratic matrix equation

$$
W^{2}-M W-J^{2}=0
$$

with the additional condition $W^{T} W=J^{2}$. If $v$ is an eigenvalue of $W$ then

$$
Q(v):=\operatorname{det}\left(v^{2} I-v M-J^{2}\right)=0 .
$$

Comparing with (13) we see that $Q(v)=v^{2 N} P\left(v^{-1}\right)$. Since $0 \notin \Sigma$ the splitting $\Sigma=\Sigma_{+}$ $\cup \Sigma_{-}$defines the splitting $S=S_{+} \cup S_{-}$of the set $S$ of roots (21):

$$
S_{+}=\left(\Sigma_{+}\right)^{-1}, \quad S_{-}=\left(\Sigma_{-}\right)^{-1} \text {. }
$$

We require that this splitting satisfies the following conditions:

$$
\bar{S}_{+}=S_{+}, \quad \bar{S}_{-}=S_{-}, \quad S_{-}=-S_{+}, \quad S_{+} \cap S_{-}=\emptyset .
$$

Such splitting exists if (21) has no purely imaginary roots. Notice that now we do allow multiple roots but we do suppose that no root belongs to both components $S_{+}$and $S_{-}$. In particular, purely imaginary roots are excluded.

We formulate Theorem 1 in the equivalent form:

Theorem 1'. For any splitting $S=S_{+} \cup S_{-}$with the properties (22) there exists a unique solution $W$ of (20) (and therefore the solution of (6) $\omega=J W^{-1}$ ) with $\operatorname{spec} W=S_{+}$.

For the proof the solution of $(20)$ will be played back to a problem of symplectic geometry, namely the determination of invariant subspaces of a linear Hamiltonian vector field.

The real $2 N \times 2 N$ matrix in question is

$$
A=\left(\begin{array}{cc}
0 & I \\
J^{2} & M
\end{array}\right)
$$

We look for an $N$-dimensional invariant subspace of $A$ :

$$
z=\left(\begin{array}{l}
X \\
Y
\end{array}\right) u, \quad u \in \mathbb{R}^{N}
$$

$X, Y$ being $N \times N$ matrices, i.e.

$$
A\left(\begin{array}{l}
X \\
Y
\end{array}\right)=\left(\begin{array}{l}
X \\
Y
\end{array}\right) C
$$

with some real $N \times N$ matrix $C$, with $\operatorname{spec} C=S_{+}$, or equivalently

$$
\left\{\begin{array}{l}
Y=X C \\
J^{2} X+M Y=Y C
\end{array} .\right.
$$

If

$$
\operatorname{det} X \neq 0 \text {, }
$$
i.e. if the invariant subspace can be viewed as the graph $y=Y X^{-1} x, z=\left(\begin{array}{l}x \\ y\end{array}\right)$ then
we obtain for $W=Y X^{-1}$,

$$
J^{2}+M W=W X C X^{-1}=W^{2},
$$

Eq. (20). 
To prove that $W^{T} W=J^{2}$, we note that $A$ is antisymmetric with respect to the symplectic bilinear form

$$
[z, w]=(B z, w), \quad B=\left(\begin{array}{cc}
M & -I \\
I & O
\end{array}\right)
$$

and $A z$ can be viewed as the Hamiltonian vector field with Hamiltonian

$$
\mathscr{H}=\frac{1}{2}(H z, z)=\frac{1}{2}\left(-|J x|^{2}+|y|^{2}\right), \quad H=\left(\begin{array}{cc}
-J^{2} & O \\
0 & I
\end{array}\right),
$$

since $B A=H$ and

$$
\dot{z}=B^{-1} \mathscr{H}_{z}=B^{-1} H z=A z
$$

Since

$$
v I-A=\left(\begin{array}{cc}
v I & O \\
-J^{2} & v^{-1} I
\end{array}\right)\left(\begin{array}{cc}
I & -v^{-1} I \\
O & v^{2} I-v M-J^{2}
\end{array}\right)
$$

we have

$$
\operatorname{det}(v I-A)=Q(v)=\operatorname{det}\left(v^{2} I-v M-J^{2}\right),
$$

and the spectrum of $A$ is $S_{+} \cup S_{-}$.

Denote the $N$-dimensional eigenspaces of $A$ with respect to $S_{+}, S_{-}$by $V_{+}, V_{-}$, respectively. Because of $\bar{S}_{+}=S_{+}, \bar{S}_{-}=S_{-}$they are real and since $\mu_{i}+\mu_{j} \neq 0$ for $\mu_{i}$, $\mu_{j} \in S_{+}$they are Lagrangian, isotropic spaces with respect to the symplectic form $[$,$] and the symmetric form \mathscr{H}$ respectively, as follows from the following lemma.

Lemma. If $E_{\mu}^{k}=\operatorname{Ker}(A-\mu I)^{k}$ and $\mu+v \neq 0$ then $\left[E_{\mu}^{k}, E_{v}^{l}\right]=0$ for all $k, l \geqq 0$.

Proof. By induction on $k+l$. For $k+l=0$ it is trivial and we assume the lemma for smaller values of $k+l$. Consider $\phi \in E_{\mu}^{k}, \psi \in E_{v}^{l}$ and set

$$
\hat{\phi}=(A-\mu I) \phi \in E_{\mu}^{k-1}, \quad \hat{\psi}=(A-v I) \psi \in E_{v}^{l-1},
$$

so that

$$
\mu \phi=A \phi-\hat{\phi}, \quad \nu \psi=A \psi-\hat{\psi},
$$

therefore

$$
(\mu+v)[\phi, \psi]=[A \phi-\hat{\phi}, \psi]+[\phi, A \psi-\hat{\psi}]=-[\hat{\phi}, \psi]-[\phi, \hat{\psi}]=0,
$$

hence $[\phi, \psi]=0$.

Corollary. The subspaces $V_{+}, V_{-}$are Lagrangian:

$$
\left[V_{+}, V_{+}\right]=\left[V_{-}, V_{-}\right]=0
$$

and isotropic with respect to $\mathscr{H}: \mathscr{H}(z)=\frac{1}{2}(H z, z)=0$ for $z \in V_{ \pm}$.

The first statement follows immediately from the lemma because

$$
V_{+}=\operatorname{span}_{s=1, \ldots, N} E_{\mu_{s}}, \quad \mu_{s} \in S_{+}, \quad E_{\mu}=\operatorname{Ker}(A-\mu I)^{2 N} .
$$

To prove that $V_{ \pm}$is isotropic with respect to $\mathscr{H}$ consider for $\phi \in E_{\mu}, \psi \in E_{v}$,

$$
(H \phi, \psi)=[A \phi, \psi]=\mu[\phi, \psi]+[\hat{\phi}, \psi]=0
$$

where $\hat{\phi}=(A-\mu I) \phi \in E_{\mu}$. 
Note that $V_{+}, V_{-}$are real subspaces since $\bar{S}_{+}=S_{+}, \bar{S}_{-}=S_{-}$, while $E_{\mu}$ are generally complex.

Now we return to the proof of Theorem 2. Let $z_{1}, \ldots, z_{n}$ be any basis in $V_{+}$; combining these as column vectors of an $N \times 2 N$ matrix

$$
Z_{+}=\left(z_{1}, \ldots, z_{n}\right)=\left(\begin{array}{c}
X_{+} \\
Y_{+}
\end{array}\right)
$$

of rank $N$ we have from $A V_{+} \subset V_{+}$

$$
A Z_{+}=Z_{+} C_{+}
$$

for some real $N \times N$ matrix $C_{+}$.

To prove (23) we use the relations (26), yielding for any $u, v \in \mathbb{R}^{N}$,

$$
0=\left[Z_{+} u, Z_{+} v\right]=\left(B Z_{+} u, Z_{+} v\right)=\left(M X_{+} u-Y_{+} u, X_{+} v\right)+\left(X_{+} u, Y_{+} v\right) \text {. }
$$

Assuming $v$ is so chosen that $X_{+} v=0$, we set $u=C_{+} v$, so that $X_{+} u=X_{+} C v=Y_{+} v$ and we find from the above identity

$$
0=\left|Y_{+} v\right|^{2}
$$

i.e. $Y_{+} v=0$, hence $Z_{+} v=0$, i.e. $v=0$. Therefore $\operatorname{det} X_{+} \neq 0$, proving (23).

Thus $V_{+}$is given by $y=W_{+} x$. Since $V_{+}$lies on the zero energy surface it follows

$$
|J x|^{2}-\left|W_{+} x\right|^{2}=0
$$

for all $x \in \mathbb{R}^{N}$ proving $W_{+}^{T} W_{+}=J^{2}$, hence $\omega=J W_{+}^{-1}$ is orthogonal. Moreover $\operatorname{spec} W_{+}=S_{+}$proving Theorem $1^{\prime}$.

\subsection{The Integration of the Discrete Euler Equation}

Now we apply our results to finding the solution of (4), following the procedure which was described in the continuous case by Dubrovin $[9,10]$.

For the initial data $X_{0}, X_{1} \in O(N)$ we define $\omega_{1}=X_{1}^{T} X_{0}=X_{1}^{-1} X_{0} \in O(N)$ and $M_{1}=\omega_{1}^{T} J-J \omega_{1}$. As follows from the previous considerations Eqs. (4) define only a correspondence, but if we fix the splitting $S=S_{+} \cup S_{-}$of the roots of the polynomial $Q(v)=\left(v^{2} I-\mu M_{k}-J^{2}\right)$, which in fact does not depend on $k$, then we have a well defined mapping $f_{S_{+}, S_{-}}:\left(\omega_{k}, M_{k}\right) \rightarrow\left(\omega_{k+1}, M_{k+1}\right)$. In order to "integrate" this dynamics consider the spectral curve $\Gamma$ :

$$
\operatorname{det}\left(M+\lambda J^{2}-\mu I\right)=0, \quad M=M_{1} .
$$

We will assume that $J^{2}$ has distinct eigenvalues different from zero: $J_{i}^{2} \neq J_{j}^{2}$ for $i \neq j$, and $J_{i}^{2} \neq 0$. For generic $M, \Gamma$ has a genus $g=\frac{(N-1)(N-2)}{2}$. The eigenvector $\psi(\lambda, \mu)$

$$
\left(M+\lambda J^{2}-\mu I\right) \psi(\lambda, \mu)=0
$$

normalized by the condition

$$
\psi^{1}+\ldots+\psi^{N}=1
$$


is meromorphic on $\Gamma$ whose poles define a divisor $\mathscr{D}=\mathscr{D}_{1}+\ldots+\mathscr{D}_{g+N-1}$ (see $[10,16])$. In the points at infinity $P_{i} \in \Gamma$, where $\mu \approx \lambda J_{i}^{2}, \lambda \rightarrow \infty(i=1, \ldots, N)$ we have

$$
\psi^{i}\left(P_{j}\right)=\delta_{j}^{i}
$$

This means that $\psi^{i}(\lambda, \mu)$ is the basis of the linear space of meromorphic functions with the pole divisor $\leqq \mathscr{D}$, determined by the conditions (30).

In our case $M$ is skew symmetric, therefore $\Gamma$ has a symmetry $\sigma: \Gamma \rightarrow \Gamma, \sigma^{2}=\mathrm{id}$ :

$$
\sigma(\lambda, \mu)=(-\lambda,-\mu) \text {. }
$$

The divisor $\mathscr{D}$ also is not arbitrary because of the following proposition. Denote $\psi^{T}(-\lambda,-\mu)$ by $\psi^{*}(\lambda, \mu)$ and fix $\lambda \in \mathbb{C}$ such that the eigenvalues $\mu_{1}, \ldots, \mu_{N}$ of $M+\lambda J^{2}$ determined by (27) are distinct.

Proposition. Let $\mu^{\prime} \neq \mu$ be two distinct eigenvalues of $M+\lambda J^{2}$, then

$$
\psi^{*}\left(\lambda, \mu^{\prime}\right) \psi(\lambda, \mu)=0 .
$$

For $\mu^{\prime}=\mu$ this product is different from zero:

$$
\psi^{*}(\lambda, \mu) \psi(\lambda, \mu) \neq 0 \text {. }
$$

To prove this consider the product $\psi^{*}\left(\lambda, \mu^{\prime}\right)\left(M+\lambda J^{2}\right) \psi(\lambda, \mu)=\mu \psi^{*}\left(\lambda, \mu^{\prime}\right) \psi(\lambda, \mu)$. On the other hand

$$
\begin{aligned}
\psi^{*}\left(\lambda, \mu^{\prime}\right)\left(M+\lambda J^{2}\right) \psi(\lambda, \mu) & =-\left(\left(M-\lambda J^{2}\right) \psi\left(-\lambda,-\mu^{\prime}\right)\right)^{T} \psi(\lambda, \mu) \\
& =\mu^{\prime} \psi^{*}\left(\lambda, \mu^{\prime}\right) \psi(\lambda, \mu) .
\end{aligned}
$$

We see that if $\mu^{\prime} \neq \mu$ then $\psi^{*}\left(\lambda, \mu^{\prime}\right) \psi(\lambda, \mu)=0$. But $\psi(\lambda, \mu)$ for all possible $\mu=\mu_{1}, \ldots, \mu_{N}$ form a basis, therefore $\psi^{*}(\lambda, \mu) \psi(\lambda, \mu)$ can't be equal to zero.

Corollary. The divisor $\mathscr{D}$ of the poles of $\psi$ satisfies the equation

$$
\mathscr{D}+\sigma(\mathscr{D}) \approx B,
$$

where $B$ is the set of branch points of $\mu$ as a function of $\lambda$, and $\approx$ means linear equivalence of divisors.

This equivalence is given by the function $F(\lambda, \mu)=\psi^{*}(\lambda, \mu) \psi(\lambda, \mu)$ as follows from the proposition. Thus $\mathscr{D}$ belongs to the shifted Prym variety $P \subset J(\Gamma)$. We restrict ourselves to these considerations because the detailed discussion of the algebraicgeometric aspects of this spectral problem can be found in the literature (see [21] and references therein). The corresponding problems of real algebraic geometry are considered in [23].

Now we use the representation (19) for describing the analytic properties on $\Gamma$ of $\psi_{k}$ for arbitrary $k$.

Fix some splitting $\Sigma=\Sigma_{+} \cup \Sigma_{-}$. As follows from (19)

$$
\tilde{\psi}(\lambda, \mu)=\left(\omega_{k}-\lambda J\right) \psi_{k}(\lambda, \mu)
$$

is an eigenvalue of $M_{k+1}+\lambda J^{2}$ :

$$
\left(M_{k+1}+\lambda J^{2}\right) \tilde{\psi}(\lambda, \mu)=\mu \tilde{\psi}(\lambda, \mu) .
$$

This means that we can define $\psi_{k+1}$ as

$$
\psi_{k+1}=\left(\omega_{k}-\lambda J\right) \psi_{k} .
$$


Notice that $\psi_{k+1}$ does not satisfy Dubrovin's normalization (29) which is required only for $\psi_{1}$.

One can see from (36) that $\psi_{k+1}$ has $N$ new poles at the "infinities" $P_{1}, \ldots, P_{N}$. In order to find the new zeros consider the hyperbola $\mathscr{H}$, determined by the equation

$$
\lambda \mu=1
$$

and the intersection $\mathscr{H} \cap \Gamma$. This intersection is described by the equations $\mu=\lambda^{-1}$ and

$$
\operatorname{det}\left(M+\lambda J^{2}-\lambda^{-1} I\right)=\left(-\lambda^{-N} \operatorname{det}\left(I-\lambda M-\lambda^{2} J^{2}\right)=0\right.
$$

which coincides with (11).

So we have $2 N$ points of intersection which we denote $Q_{1}^{+}, \ldots, Q_{N}^{+}, Q_{1}^{-}, \ldots, Q_{N}^{-}$in agreement with the splitting $\Sigma=\Sigma^{+} \cup \Sigma^{-}$. As follows from the construction of $\omega_{k}$ (see Sect. 3)

$$
\left(\omega_{k}-\lambda_{i} I\right) \psi_{k}\left(Q_{i}^{+}\right)=0
$$

This means that $Q_{1}^{+}, \ldots, Q_{N}^{+}$are the new zeros of $\psi_{k+1}$. Thus we have proven

Lemma. For a given splitting $\Sigma=\Sigma_{+} \cup \Sigma_{-}$the vector eigenfunction $\psi_{k+1}$ (36) of the matrix $M_{k+1}+\lambda J^{2}$ has on the spectral curve $\Gamma$ the following analytical properties, which determine $\psi_{k+1}$ uniquely:

1. $\psi_{k+1}$ has a simple pole in $\mathscr{D}$ depending on the initial data $M_{1}$ and the poles at the "infinities" $P_{1}, \ldots, P_{N}$ with asymptotics in

$$
P_{j}: \psi_{k+1}^{i}=\lambda^{k}\left(-J_{j}\right)^{k}\left(\delta_{j}^{i}+O\left(\lambda^{-1}\right)\right), \quad \lambda \rightarrow \infty .
$$

2. $\psi_{k+1}$ has a zero of order $k$ in $Q_{1}^{+}, \ldots, Q_{N}^{+}$.

In particular, we see that the pole-divisor $\mathscr{D}_{k+1}$ of $\psi_{k+1}$ is connected with $\mathscr{D}_{k}$ by the relation

$$
\mathscr{D}_{k+1} \approx \mathscr{D}_{k}+U \text {, }
$$

where $U=P_{1}+\ldots+P_{N}-Q_{1}^{+}-\ldots-Q_{N}^{+}$.

For given $\psi_{k+1}$ one can reconstruct $\omega_{k+1}$ by using the formula (14), and $M_{k+1}$ as $\omega_{k+1}^{T} J-J \omega_{k+1}$. To find the solution of $(2) X_{k} \in O(N)$ :

$$
X_{k}^{-1}=\omega_{k} \omega_{k-1} \ldots \omega_{1} X_{0}^{-1},
$$

one can use again Eq. (36). Indeed, from (36) follows that

$$
\Phi_{k+1}=\omega_{k} \Phi_{k}
$$

where $\Phi_{k}$ is $N \times N$ matrix with $\psi_{k}\left(0, \mu_{i}\right)$ as a column. This means that

$$
\Phi_{k+1}=\omega_{k} \omega_{k-1} \ldots \omega_{1} \Phi_{1} \text { and } X_{k}^{-1}=\Phi_{k+1} \Phi_{1}^{-1} X_{0}^{-1} \text {. }
$$

For $\psi_{k+1}$ one can write the explicit formulas in terms of Prym's $\theta$-functions as it was done, for example, by Bobenko in [24]. But here we restrict ourselves to the example of $O(3)$ (see below).

We summarize the result of this section in the following theorem.

Theorem 3. The discrete Euler Eq. (4) corresponds to the shifts on the Prym variety $P \subset J(\Gamma)$ (34) by the vector $U=P_{1}+\ldots+P_{N}-Q_{1}^{+}-\ldots-Q_{N}^{+}$, depending on the splitting $\Sigma=\Sigma_{+} \cup \Sigma_{-}$. If such splitting is fixed the general solution of (4) and (2) can be expressed as some abelian function on $P$ in the points $z_{k}=z_{0}+k U$. 


\subsection{Explicit Formulas for the Discrete Dynamics} of the 3-Dimensional Rigid Body

We consider here Eqs. (2), (4) for $N=3$. In this case the solution can be expressed by elliptic functions. The spectral curve $\Gamma(27)$ has the equation

$$
\operatorname{det}\left|\begin{array}{ccc}
\lambda J_{1}^{2}-\mu & M_{12} & M_{13} \\
-M_{12} & \lambda J_{2}^{2}-\mu & M_{23} \\
-M_{13} & -M_{23} & \lambda J_{3}^{2}-\mu
\end{array}\right|=0
$$

or

$$
\left(\lambda J_{1}^{2}-\mu\right)\left(\lambda J_{2}^{2}-\mu\right)\left(\lambda J_{3}^{2}-\mu\right)+H \lambda-M^{2} \mu=0,
$$

where $H=J_{3}^{2} M_{12}^{2}+J_{2}^{2} M_{13}^{2}+J_{1}^{2} M_{23}^{2}$.

In the new variables

$$
x=\mu / \lambda, \quad y=\lambda,
$$

(41) has the form

$$
y^{2} Q(x)=H-M^{2} x
$$

with $Q(x)=\left(x-J_{1}^{2}\right)\left(x-J_{2}^{2}\right)\left(x-J_{3}^{2}\right)$. After another change of variables

$$
w=Q(x) y=Q(\mu / \lambda) \lambda
$$

we obtain the standard form of the elliptic curve

$$
w^{2}=R(x)=\left(x-J_{1}^{2}\right)\left(x-J_{2}^{2}\right)\left(x-J_{3}^{2}\right)\left(H-M^{2} x\right) .
$$

The involution $\sigma:(\lambda, \mu) \rightarrow(-\lambda,-\mu)$ in these variables is $\sigma(w, x)=(-w, x)$ and the Prym variety coincides with $J(\Gamma) \approx \Gamma$. The "infinities" $P_{1}, P_{2}, P_{3}$ correspond to the branch points $x=J_{1}^{2}, x=J_{2}^{2}, x=J_{3}^{2}$. The fourth branch point $x=H / M^{2}$ corresponds to the point $\lambda=\mu=0$, so we choose it as the zero 0 on $\Gamma$. Let $x_{1}<x_{2}$ $<x_{3}<x_{4}$ be the ordered roots of $R(x)$, i.e. the numbers $J_{1}^{2}, J_{2}^{2}, J_{3}^{2}, H / M^{2}$ (notice that $\min \left\{J_{i}\right\} \leqq H / M^{2} \leqq \max \left\{J_{i}\right\}$ ).

The elliptic integral

$$
z=\int_{H / M^{2}}^{(x, w)} \frac{d x}{\sqrt{R(x)}}
$$

gives the equivalence of $\Gamma$ and $\mathbb{C} / \mathbb{Z} \tau_{1}+\mathbb{Z} \tau_{2}$, where $\tau_{1}=2 \int_{x_{1}}^{x_{2}} \frac{d x}{\sqrt{R(x)}}$, $\tau_{2}=2 \int_{x_{2}}^{x_{3}} \frac{d x}{\sqrt{R(x)}} ; \tau_{1}$ is real, $\tau_{2}$ is purely imaginary.

The equation $\mu \lambda=1$ in the variables $x, w$ has the form

$$
\left(x-J_{1}^{2}\right)\left(x-J_{x}^{2}\right)\left(x-J_{3}^{2}\right)-x\left(H-M^{2} x\right)=0 ;
$$

it determines on $\Gamma$ the set of six points, which we denote as $Q_{1}^{+}, Q_{2}^{+}, Q_{3}^{+}, Q_{1}^{-}, Q_{2}^{-}$, $Q_{3}^{-}$according to the splitting $\Sigma=\Sigma_{+} \cup \Sigma_{-}$(see Fig. 1)

This figure depicts the situation, corresponding to $J_{1}^{2}<H / M^{2}<J_{2}^{2}<J_{3}^{2}$ and sufficiently small $M^{2}$ and $H$, when all roots of $(43)$ and $P(\lambda)$ are real numbers. The condition that $P(\lambda)$ has no purely imaginary roots, which is sufficient for the 


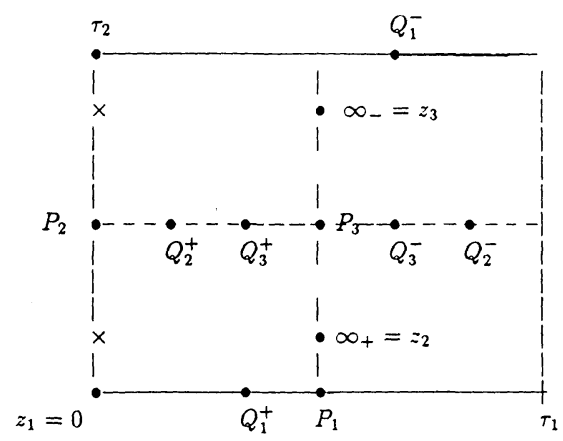

Fig. 1

solvability of Eq. (6) (see Sects. 1.2 and 1.4), is equivalent to the absence of negative roots for Eq. (43). This leads to a certain restriction on the integrals $M^{2}$ and $H$. The shift $U$ is given by

$$
U=P_{1}+P_{2}+P_{3}-Q_{1}^{+}-Q_{2}^{+}-Q_{3}^{+}=-\left(Q_{1}^{+}+Q_{2}^{+}+Q_{3}^{+}\right),
$$

because of $P_{1}+P_{2}+P_{3}=0$.

Using the results of the previous section one can easily express $\psi_{k+1}(z)$ in terms of the classical elliptic $\sigma$-function and the initial position of the poles $\psi_{1}:\left(\zeta_{1}, \zeta_{2}, \zeta_{3}\right)$. For example, the function

$$
\begin{aligned}
& \psi_{k+1}^{1}(z) \\
& =\left(-J_{1}\right)^{k} \frac{\sigma^{k}\left(z-Q_{1}^{+}\right) \sigma^{k}\left(z-Q_{2}^{+}\right) \sigma^{k}\left(z-Q_{3}^{+}\right) \sigma\left(z-\left(\zeta_{1}+\zeta_{2}+\zeta_{3}\right)-k U-P_{1}\right)}{\sigma\left(z-\zeta_{1}\right) \sigma\left(z-\zeta_{2}\right) \sigma\left(z-\zeta_{3}\right) \sigma^{k}\left(z-P_{1}\right) \sigma^{k-1}\left(z-P_{2}\right) \sigma^{k-1}\left(z-P_{3}\right)}
\end{aligned}
$$

has all analytic properties of the first component of $\psi_{k+1}(z)$ (see the lemma in Sect. 1.5) and therefore coincides with it. We can write now explicit formulas for $\omega_{k}$, $M_{k}$, and $X_{k}$ as it was explained in the previous section.

Omitting nonessential multipliers in (44) we define a matrix $\tilde{\Phi}_{k+1}$ as

$$
\left(\tilde{\Phi}_{k+1}\right)_{i j}=\left(-J_{i}\right)^{k} f^{k}\left(z_{j}\right) \frac{\sigma\left(z_{j}-\zeta-k U-P_{i}\right)}{\sigma\left(z_{j}-P_{i}\right)},
$$

where

$$
f(z)=\frac{\sigma\left(z-Q_{1}^{+}\right) \sigma\left(z-Q_{2}^{+}\right) \sigma\left(z-Q_{3}^{+}\right)}{\sigma\left(z-P_{1}\right) \sigma\left(z-P_{2}\right) \sigma\left(z-P_{3}\right)},
$$

$\zeta=\zeta_{1}+\zeta_{2}+\zeta_{3}, z_{i}(i=1,2,3)$ correspond to $\lambda=0$ in (41):

$$
z_{i}=0\left(x=\frac{H}{M^{2}}\right), \quad z_{2,3}= \pm \int_{H / M^{2}}^{\infty} \frac{d x}{\sqrt{R(x)}} \quad(x=\infty) \text {. }
$$

Finally we have from (40)

$$
X_{k}^{T}=\widetilde{\Phi}_{k+1} \tilde{\Phi}_{1}^{-1} X_{0}^{T}, \quad \omega_{k}=\tilde{\Phi}_{k+1} \tilde{\Phi}_{k}^{-1}
$$

with $\widetilde{\Phi}_{k}$ defined by (45). 
In the continuous limit for $M=\varepsilon M_{c}, \varepsilon \rightarrow 0$ the curve $\Gamma$ remains

$$
\left(w^{\prime}\right)^{2}=\left(\frac{w}{\varepsilon}\right)^{2}=\left(x-J_{1}^{2}\right)\left(x-J_{2}^{2}\right)\left(x-J_{3}^{2}\right)\left(H_{c}-M_{c}^{2} x\right) s,
$$

but Eq. (43) becomes

$$
\left(x-J_{1}^{2}\right)\left(x-J_{2}^{2}\right)\left(x-J_{3}^{2}\right)-\varepsilon^{2} x\left(H_{c}-M_{c}^{2} x\right)=0 .
$$

When $\varepsilon=0$ we arrive at

$$
\left(x-J_{1}^{2}\right)\left(x-J_{2}^{2}\right)\left(x-J_{3}^{2}\right)=0 .
$$

This means that $Q_{1}^{ \pm}, Q_{2}^{ \pm}, Q_{3}^{ \pm}$tend to $P_{1}, P_{2}, P_{3}$ when $\varepsilon \rightarrow 0$ and the shift $U=\Sigma Q_{i}$ $-\Sigma Q_{i}^{+} \rightarrow 0$. One should observe that the continuous limit corresponds to the special splitting $\Sigma$, where: $\Sigma_{+}$contains all roots in the right half plane of $P(\lambda)$ of $(13)$ Sect. 1.2, which becomes here

$$
\left(\lambda^{2} J_{1}^{2}-1\right)\left(\lambda^{2} J_{2}^{2}-1\right)\left(\lambda^{2} J_{3}^{2}-1\right)+H \lambda^{4}-M \lambda^{2}=0 .
$$

As was shown before this continuous limit coincides with the classical problem about the force free motion of a rigid body, the explicit solution for which in terms of elliptic functions were found by Jacobi [25]. The comparison of this formula and the continuous limit of (46) may be complicated.

\section{The Discrete Dynamics on Stiefel Manifolds and the Heisenberg Chain with Classical Spins}

In this section we generalize the previous results and consider the functional $S=\sum_{k} \operatorname{tr}\left(X_{k} J X_{k+1}\right)$ on the sequences $X=\left(X_{k}\right)$ of $n+N$ matrices $X_{k}, 1 \leqq n \leqq N$ satisfying the condition

$$
X_{k} X_{k}^{T}=I_{n} .
$$

The rows of such matrices are orthogonal unit vectors in $\mathbb{R}^{N}$. Such matrices form the Stiefel manifold $V_{n, N}$. The function of the interaction $\mathscr{L}=\operatorname{tr}\left(X J Y^{T}\right)$ is a symmetric bilinear function, invariant under the action of $O(n): X \rightarrow s X, Y \rightarrow s Y$, $s \in O(n)$. We show that some results of Sect. 1, which correspond to the case $n=N$, can be generalized for $n<N$.

For $n=1$ our results agree with the solution of this problem proposed in [1]. Notice that for $n=1, N=3$ we have the problem about the stationary states of a Heisenberg chain with classical spins (see [1, 5-7] and Introduction).

\subsection{The Equation of the Dynamics and Isospectral Deformations}

The variation of $S$ under the condition (1) leads to the equation

$$
X_{k+1} J+X_{k-1} J=\Lambda_{k} X_{k},
$$

where $\Lambda_{k}=\Lambda_{k}^{T}$ is an $(n \times n)$ matrix multiplier. We assume $J$ to be symmetric and nondegenerate.

Theorem 4. The Eqs. (2) are equivalent to the equation of isospectral deformations

$$
L_{k+1}(\lambda)=A_{k}(\lambda) L_{k}(\lambda) A_{k}^{-1}(\lambda)
$$


with $L_{k}(\lambda)=J^{2}+\lambda M_{k}-\lambda^{2} X_{k-1}^{T} X_{k-1}, M_{k}=X_{k-1}^{T} X_{k} J-J X_{k}^{T} X_{k-1}$, $A_{k}(\lambda)=J-\lambda X_{k}^{T} X_{k-1}$. Moreover,

$$
L_{k}=\left(J+\lambda X_{k-1}^{T} X_{k}\right)\left(J-\lambda X_{k}^{T} X_{k-1}\right)=A_{k}^{T}(-\lambda) A_{k}(\lambda)
$$

so that

$$
L_{k+1}=A_{k+1}^{T}(-\lambda) A_{k+1}(\lambda)=A_{k}(\lambda) A_{k}^{T}(-\lambda) .
$$

The proof follows by a direct calculation. The determinant of $L_{k}$ has the form

$$
\begin{aligned}
P(\lambda) & =\operatorname{det} L_{k}(\lambda)=\operatorname{det} A_{k}^{T}(-\lambda) \operatorname{det} A_{k}(\lambda) \\
& =\operatorname{det} J^{2} \operatorname{det}\left(I_{n}-\lambda^{2}\left(X_{k-1} J^{-1} X_{k}\right)^{2}\right),
\end{aligned}
$$

where we used the Weinstein-Aronszajn formula, see [10]. Therefore $P(\lambda)$ is an even polynomial in $\lambda$ of degree $2 n$. The factorization (4) determines the splitting $\Sigma=\Sigma_{+} \cup \Sigma_{-}$of the set $\Sigma$ of zeros of $P(\lambda): \Sigma_{+}=\{\lambda: \operatorname{det} A(\lambda)=0\}$, $\Sigma_{-}=\left\{\lambda: \operatorname{det} A^{T}(-\lambda)=0\right\}$. This splitting satisfies the conditions

$$
\bar{\Sigma}_{+}=\Sigma_{+}, \quad \bar{\Sigma}_{-}=\Sigma_{-}, \quad \Sigma_{+}=-\Sigma_{-} .
$$

If such a splitting is fixed and all zeros of $P(\lambda)$ are distinct then for given $X_{k-1}, X_{k}$ the matrix $X_{k+1}$ can be defined as follows. $\lambda_{i} \in \Sigma_{+}$,

Consider the "eigenvectors" $\psi_{i}$ of $L_{k+1}=A_{k}(\lambda) A_{k}^{T}(-\lambda)$, corresponding to

$$
L_{k+1}\left(\lambda_{i}\right) \psi_{i}=0
$$

and combine these vectors to an $N \times n$ matrix $\psi$ as columns. Then because $\Sigma_{+}=\left\{\lambda: \operatorname{det} A_{k+1}(\lambda)=0\right\}$ we have from (5),

$$
\left(J-\lambda_{i} X_{k+1}^{T} X_{k}\right) \psi_{i}=0
$$

or

$$
J \psi-X_{k+1}^{T} X_{k} \psi \Lambda=0
$$

with $\Lambda=\operatorname{diag}\left(\lambda_{1}, \ldots, \lambda_{n}\right), \lambda_{i} \in \Sigma_{+}$. If the $n \times n$ matrix $X_{k} \psi$ is invertible then

$$
X_{k+1}^{T}=J \psi \Lambda^{-1}\left(X_{k} \psi\right)^{-1}
$$

is reconstructed uniquely.

Leaving the further discussion of the general case $1 \leqq n<N$ we now turn to a detailed treatment of the case $n=1$ in the next section. In this connection we want to mention the work of Adams, Harnad, and Previato [26], in which the matrices introduced in [10] are generalized.

\subsection{Discrete Version of the Neumann System and the Heisenberg Chain With Classical Spins}

For $n=1$ we have the functional

$$
S(x)=\sum_{k \in \mathbb{Z}}\left(x_{k}, J x_{k+1}\right),
$$

where $x=\left(x_{k}\right), x_{k}$ belong to the unit sphere $S^{N-1}$ in $\mathbb{R}^{N}:\left|x_{k}\right|=1$ and $J=$ $\operatorname{diag}\left(J_{1}, \ldots, J_{n}\right)$. For $N=3$ we have unit vectors in $\mathbb{R}^{3}$ which can be interpreted as 
classical spins. In this case the functional $S(10)$ defines the energy of a spin chain in the Heisenberg model (see [1, 6-8]). The equations of the stationary configuration have the form

$$
J x_{k+1}+J x_{k-1}=\lambda_{k} x_{k}
$$

or

$$
x_{k+1}+x_{k-1}=\lambda_{k} J^{-1} x_{k} .
$$

The multipliers $\lambda_{k}(1 \times 1$ matrix $)$ can be found from

$$
1=\left|x_{k+1}\right|^{2}=\left|\lambda_{k} J^{-1} x_{k}-x_{k-1}\right|^{2}=\lambda_{k}^{2}\left|J^{-1} x_{k}\right|^{2}-2 \lambda_{k}\left(J^{-1} x_{k}, x_{k-1}\right)+1,
$$

therefore

$$
\lambda_{k}\left(\lambda_{k}\left|J^{-1} x_{k}\right|^{2}-2\left(J^{-1} x_{k}, x_{k-1}\right)\right)=0 .
$$

We have two possibilities: $\lambda_{k}=0$ or

$$
\lambda_{k}=2\left(J^{-1} x_{k}, x_{k-1}\right) /\left.|| J^{-1} x_{k}\right|^{2} .
$$

In the literature (see, for example, $[5,6]$ ) usually the second possibility is considered because only in this case it is possible to have the continuous limit. This can be seen as follows. Let $J=I+\varepsilon^{2} J_{c}$ and $x_{k}=x\left(t_{0}+k \varepsilon\right)$ for small $\varepsilon$, then from (11) we have for $x(t)$ the equations

$$
\left(I+\varepsilon^{2} J_{c}\right)\left(2 x+2 \frac{1}{2} \varepsilon^{2} x^{\prime \prime}\right) \approx \lambda x
$$

or

$$
x^{\prime \prime}+2 J_{c} x \approx \mu x
$$

with $\mu=(\lambda-2) \varepsilon^{-2}$, i.e. the Neumann system describing the motion on the unit sphere under the influence of the potential $U(x)=\left(J_{c} x, x\right)[8,9,27]$. For this reason we call the system (11), (13) a discrete version of the Neumann system.

Now we explain how this system can be integrated with the help of Theorem 4. The matrix $L_{k}(3)$ has the form

$$
L=J^{2}+\lambda x \wedge J y-\lambda^{2} x \otimes x,
$$

where $x=x_{k-1}, y=x_{k}, x \wedge y=x \otimes y-y \otimes x$. This is a special case of the matrix introduced and investigated in connection with the classical integrable systems in [10]. The determinant $L$ has the form

$$
\operatorname{det} L=\operatorname{det} J^{2}\left(1-\lambda^{2}\left(x, J^{-1} y\right)^{2}\right)
$$

and the zeros $\lambda= \pm\left(x, J^{-1} y\right)^{-1}$. So we have two possible splittings of this set $\Sigma=\Sigma_{+} \cup \Sigma_{-}: \Sigma_{+}=\left(x, J^{-1} y\right)^{-1}$ or $\Sigma_{+}=-\left(x, J^{-1} y\right)^{-1}$. It is easy to see that they correspond to two solutions of (12). This means that the procedure proposed in the previous section actually determines the dynamics.

In order to get explicit formulas we have to consider the spectral curve $\Gamma$ of $L$ :

$$
\operatorname{det}(L(\lambda)-\mu I)=0,
$$

or using the formula (see [10])

$$
\frac{\operatorname{det}(L-\mu I)}{\operatorname{det}\left(J^{2}-\mu I\right)}=1-\lambda^{2} \phi_{\mu}(x, J y),
$$


$\phi_{\mu}(x, J y)=Q_{\mu}(x)-\left(Q_{\mu}(x) Q_{\mu}(J y)-Q_{\mu}^{2}(x, J y)\right), \quad Q_{\mu}(x, z)=\left(-\left(J^{2}-\mu I\right)^{-1} x, z\right), \quad Q_{\mu}(x)$ $=Q_{\mu}(x, x)$, we obtain the hyperelliptic equation

$$
\lambda^{2}\left[\left(Q_{\mu}(x) Q_{\mu}(J y)-Q_{\mu}^{2}(x, J y)\right)-Q_{\mu}(x)\right]+1=0 .
$$

The function $\dot{\varphi}_{\mu}(x, J y)$ can be represented as

$$
\varphi_{\mu}(x, J y)=\sum_{i=1}^{N} \frac{F_{i}(x, y)}{\mu-J_{i}^{2}}
$$

where

$$
F_{i}=x_{i}^{2}+\sum_{j \neq i} \frac{(x \wedge J y)_{i j}^{2}}{J_{i}^{2}-J_{j}^{2}}, \quad \sum_{i=1}^{N} F_{i} \equiv 1
$$

are the involutive integrals of the system (11), (13) (see $[1,8])$. Denoting the roots of $\varphi_{\mu}(x, J y)$ by $\mu_{1}, \ldots, \mu_{N-1}$ we can write

$$
\varphi_{\mu}(x, J y)=\frac{\prod_{i=1}^{N-1}\left(\mu-\mu_{i}\right)}{\prod_{i=1}^{N}\left(\mu-J_{i}^{2}\right)}
$$

and the equation of $\Gamma$ as

$$
v^{2}=\prod_{i=1}^{N-1}\left(\mu-\mu_{i}\right) \prod_{j=1}^{N}\left(\mu-J_{j}^{2}\right)
$$

where $v=\lambda \prod_{i=1}^{N-1}\left(\mu-\mu_{i}\right)$. We see that the mapping (11) is the shift on the Jacobi variety of the hyperelliptic curve (23). To find this shift we follow the same procedure as in Sect. 1.5; namely the eigenfunction $\psi_{k+1}$ of the matrix $L_{k+1}$ can be obtained as $\psi_{k+1}=A_{k}(\lambda) \psi_{k}$ or

$$
\psi_{k+1}=\left(J-\lambda x_{k} \otimes x_{k-1}\right) \psi_{k} .
$$

We see that $\psi_{k+1}$ has a new pole at "infinity" $P_{\infty}$ corresponding to $\mu=\infty$ for (23) and a new zero in one of the points $P_{ \pm}: \mu=0, \lambda= \pm\left(x, J^{-1} y\right)^{-1}$, determined by the splitting $\Sigma=\Sigma_{+} \cup \Sigma_{-}$. So the shift is $U=P_{\infty}-P_{+}$. Following the procedure proposed in the previous paragraph one can write the explicit formulas for $\psi_{k}$ and $x_{k}$ in terms of hyperelliptic $\theta$-functions. Such formulas were found first in [7] (see also [1]) by another approach, requiring some unmotivated steps. These results are in a good agreement with those of $[1,7]$, as one can verify.

\section{The Billiard Inside an Ellipsoid}

Now we apply the above procedure to describe the billiard motion inside a domain $\Omega \subset \mathbb{R}^{N}$ bounded by the ellipsoid $Q=\partial \Omega$ given by the equation

$$
(A x, x)=1 \text {, }
$$

where $A$ is a positive symmetric $N$ by $N$ matrix. This application has some surprising features. In particular, it leads in a natural way to a mapping $\phi$ which is not directly related to the system under consideration while $\phi \circ \phi=\phi^{2}$ is. In other 
words this mapping can be viewed as a "square root" of the billiard mapping. Moreover, this mapping $\phi$ commutes with the billiard mapping, hence takes a billiard orbit into another billiard orbit. Such a symmetry of the billiard problem was found in [1]. We begin with the construction of this mapping $\phi$.

The dynamics of the billiard in the domain $\Omega$ can be described as the stationary points of the functional $S$ :

$$
S=\sum_{k \in \mathbb{Z}}\left|x_{k}-x_{k-1}\right|, \quad x_{k} \in Q
$$

The equation of motion can be written in the form (see Fig. 2):

$$
\left\{\begin{array}{l}
x_{k+1}-x_{k}=\mu_{k} y_{k+1} \\
y_{k+1}-y_{k}=v_{k} A x_{k}
\end{array},\right.
$$

where $y_{k}=\left(x_{k}-x_{k-1}\right) /\left|x_{k}-x_{k-1}\right|,\left|y_{k}\right|=1$ be the momentum, the multipliers $\mu_{k}, v_{k}$ are determined from the conditions $\left|y_{k}\right|=1,\left(A x_{k}, x_{k}\right)=1$ :

$$
\mu_{k}=-\frac{2\left(A y_{k+1}, x_{k}\right)}{\left(A y_{k+1}, y_{k+1}\right)}, \quad v_{k}=-\frac{2\left(A x_{k}, y_{k}\right)}{\left(A x_{k}, A x_{k}\right)} \text {. }
$$

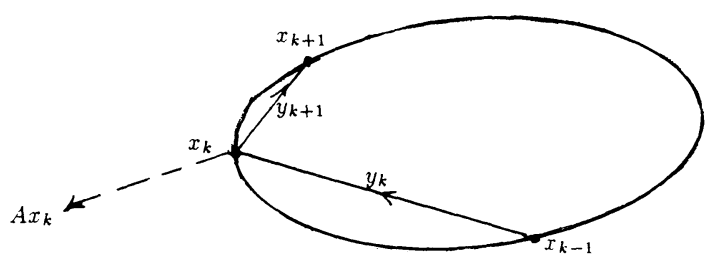

Fig. 2

Consider the "skew-hodograph" mapping $\phi:(x, y) \rightarrow\left(x^{\prime}, y^{\prime}\right)$ determined by the formulas

$$
\left\{\begin{array}{l}
x_{k}^{\prime}=C y_{k+1}=C\left(y_{k}+v_{k} A x_{k}\right) \\
y_{k}^{\prime}=-C^{-1} x_{k}
\end{array}\right.
$$

with $C=A^{-1 / 2}$ (compare with [1]). One can easily check that if $\left(x_{k}, y_{k}\right)$ is a solution of (3), so is $\left(x_{k}^{\prime}, y_{k}^{\prime}\right)$. Moreover, $x_{k}^{\prime \prime}=C y_{k+1}^{\prime}=-x_{k+1}, y_{k}^{\prime \prime}=-C^{-1} x_{k}^{\prime}=-y_{k+1}$, showing that the dynamics of $\phi$ "contains" the billiard dynamics.

In the continuous limit this symmetry means only that if $x(s)$ is a geodesic on the ellipsoid (1) parametrized by the length then the trajectory of the vector $\tilde{x}(s)$ $=C \dot{x}(s)$ is also a geodesic line on the same ellipsoid (but in general $s$ is not the length of $\tilde{x}(s)$ ). This geometrical fact was observed in [28]. Notice that the operator $C$ transforms the unit sphere into the ellipsoid $Q$, whose equation can be rewritten as

$$
\left(C^{-2} x, x\right)=\left|C^{-1} x\right|^{2}=1
$$

\subsection{The Splittings and Isospectral Deformations}

Consider the matrix $L(x, y, \lambda)$ given by

$$
L=A^{-1}-\lambda x \wedge y-\lambda^{2} y \otimes y
$$


which was proposed in connection with the geodesic flow on the ellipsoid in $[10,9]$. For $x \in Q$ this matrix $L$ can be factored:

$$
L(x, y, \lambda)=(C+\lambda y \otimes \xi)(C-\lambda \xi \otimes y)
$$

with $\xi=C^{-1} x,|\xi|=1$. If $x=x_{k}, y=y_{k}$ then (6) corresponds to the splitting $\Sigma=\{\lambda: \operatorname{det} L=0\}=\Sigma_{+} \cup \Sigma_{-}$into the positive and negative part correspondingly. Indeed, it is easy to show (see [10]) that for $x \in Q,|y|=1$,

$$
\operatorname{det} L=\operatorname{det} A^{-1}\left(1-\lambda^{2}(A x, y)^{2}\right)
$$

and $\Sigma_{+}=\{\lambda: \operatorname{det}(C-\lambda \xi \otimes y)=0\}=\left\{\left(C^{-1} \xi, y\right)^{-1}\right\}=\left\{\left(C^{-2} x, y\right)^{-1}\right\}=\left\{(A x, y)^{-1}\right\}$. But $\left(A x_{k}, y_{k}\right)>0$ (see Fig. 2) therefore in this case $\Sigma_{+}$consists of the positive root of (7). We will call such splitting natural.

Together with such splitting of $L(x, y, \lambda)$

$$
L=(C+\lambda y \otimes \xi)(C-\lambda \xi \otimes y), \quad \xi=C^{-1} x, \quad|\xi|=|y|=1,
$$

we consider the matrix $L^{\prime}(x, y, \lambda)$ obtained by exchanging the factors:

$$
L^{\prime}=(C-\lambda \xi \otimes y)(C+\lambda y \otimes \xi),
$$

and split it again in the natural way

$$
L^{\prime}=\left(C+\lambda y^{\prime} \otimes \xi^{\prime}\right)\left(C-\lambda \xi^{\prime} \otimes y^{\prime}\right), \quad\left|\xi^{\prime}\right|=\left|y^{\prime}\right|=1 .
$$

Theorem 5. The mapping $(x, y) \rightarrow\left(x^{\prime}=C \xi^{\prime}, y^{\prime}=-\xi\right)$ determined by (8), (9) and describing the isospectral deformations of the matrix $L \rightarrow L^{\prime}$, coincides with the mapping $\phi$ of (4).

Proof. The second equation $y^{\prime}=-\xi=-C^{-1} x$ coincides with that of (4). In order to prove the same for the first equation compare (8) and (9) with $y^{\prime}=-\xi$ : $C^{2}-\lambda \xi \wedge C y-\lambda^{2} \xi \otimes \xi=C^{2}+\lambda C \xi^{\prime} \wedge \xi-\lambda^{2} \xi \otimes \xi\left(\xi^{\prime}, \xi^{\prime}\right)$. We see that $\xi \wedge\left(C y-C \xi^{\prime}\right)$ $=0$ or

$$
\xi^{\prime}-y=\alpha C^{-1} \xi
$$

for some $\alpha \in \mathbb{R},\left|\xi^{\prime}\right|^{2}=1$. But $\left|\xi^{\prime}\right|^{2}=\left|y+\alpha C^{-1} \xi\right|^{2}=|y|^{2}+2 \alpha\left(C^{-1} \xi, y\right)+\alpha^{2}\left|C^{-1} \xi\right|^{2}$ and therefore we have two possibilities $\alpha=0$ and $\alpha=-\frac{2\left(C^{-1} \xi, y\right)}{\left|C^{-1} \xi\right|^{2}}=-\frac{2(A x, y)}{(A x, A x)}$. One can easily check that the second possibility corresponds to the natural splitting and leads to the first equation of (4).

The explicit formulas for the ellipsoidal billiard were found in [1] by using the connection with the spectral theory of the difference operator. Now we are also able to incorporate these formulas into the framework of Sect. 2.

Corollary. If $\left(x_{k}, y_{k}\right)$ is the solution of the billiard system (3) then the matrices $L_{k}=L\left(x_{k}, y_{k}, \lambda\right)$ satisfy the equation

$$
L_{k+1}=A_{k} L_{k} A_{k}^{-1}
$$

with $A_{k}=A^{-1}-\lambda\left(x_{k} \otimes y_{k}-y_{k+1} \otimes x_{k}\right)-\lambda^{2} y_{k+1} \otimes y_{k}$.

Indeed,

$$
\begin{aligned}
L_{k+1} & =L^{\prime \prime}=\left(C+\lambda \xi^{\prime} \otimes \xi\right) L^{\prime}\left(C+\lambda \xi^{\prime} \otimes \xi\right)^{-1} \\
& =\left(C+\lambda \xi^{\prime} \otimes \xi\right)(C-\lambda \xi \otimes y) L_{k}(C-\lambda \xi \otimes y)^{-1}\left(C+\lambda \xi^{\prime} \otimes \xi\right)^{-1} \\
& =A_{k} L_{k} A_{k}^{-1}
\end{aligned}
$$


since $\xi^{\prime}=y_{k+1}, \xi=-y_{k}, C \xi=x_{k}$. Clearly, (11) can also be checked by a direct calculation.

\subsection{Connection Between the Ellipsoidal Billiard and the Discrete Neumann System}

Replacing $x_{k}^{\prime}, y_{k}^{\prime}$ by $x_{k+1}, y_{k+1}$ we rewrite the system $\phi$ of (4) as

$$
\left\{\begin{array}{l}
x_{k+1}=C y_{k}+v_{k} C^{-1} x_{k} \\
y_{k+1}=-C^{-1} x_{k}
\end{array}\right.
$$

or as

$$
x_{k+1}+x_{k-1}=v_{k} C^{-1} x_{k} .
$$

Denoting $C^{-1} x_{k}=q_{k},\left|q_{k}\right|=1$ we have

$$
q_{k+1}+q_{k-1}=v_{k} C^{-1} q_{k}, \quad\left|q_{k}\right|=1
$$

which coincides with (11) of Sect. 2.2 with $J=C$.

Theorem 6. If $\left(x_{k}, y_{k}\right)$ is a solution of the system (4'), connected with the ellipsoidal billiard (1) as explained before, then $q_{k}=C^{-1} x_{k}$ is the solution of the discrete version of the Neumann system ([12], Sect. 2) with $J=C$. Conversely, if $q_{k}$ is the solution of (13), then $x_{k}=C q_{2 k}(-1)^{k}$ is a trajectory of the billiard's point in the ellipsoid (1) with $A=J^{-2}$.

This theorem, reminiscent of the connection between the geodesic flow and the Neumann system of [9], can, however, not be considered as a "discretization" of Knörrer's result [16]. First of all instead of the Gaussian mapping we have a linear mapping $C$. The second new feature is the appearance of the mapping $\phi$. Moreover, this connection is unexpected because of the different character of the transition to the continuous limit for this discrete system: for the billiard it corresponds simply to the dynamics near the diagonal in $Q \times Q$, but for the system (11), Sect. 2.2, the diagonal is not invariant, so we have to use another limit process. There is another possibility in considering the dynamics near the zero level of the integral $F=\left(J^{-1} x_{k}, x_{k-1}\right)$ which corresponds to $\lambda_{k} \approx 0, x_{k+1}+x_{k-1} \approx 0$ [see formulas (5), (6), Sect. 2]. This possibility leads to the ellipsoidal billiard, as follows from Theorem 6 .

Remark. A natural question arises whether or not the solutions for our discrete systems have the form $x_{k}=x(k \Delta)$, where $x(t)$ is a solution of the corresponding classical problem. The answer is: in general not. This can be seen in the example of the ellipsoidal billiard. Suppose that every orbit lies on one geodesic. Fix $q_{0}$ on a symmetry plane $O x y: q_{0}=\left(x_{0}, y_{0}, 0\right), x_{0}: y_{0} \neq 0$, and choose $q_{-1}$ on the intersection of the ellipsoid with the plane, orthogonal to $O x y$ and containing the normal to the ellipsoid in $q_{0}$. Obviously $q_{1}=\sigma\left(q_{-1}\right), \sigma(x, y, z)=(x, y,-z)$. For $q_{0}$ and $q_{-1}$ being close there exists a unique geodesic line $\gamma$, passing through $q_{0}$ and $q_{-1}$. From our assumption it follows that it passes also through $q_{1}$. From the uniqueness $\sigma(\gamma)=\gamma$ and therefore the tangent to $\gamma$ in $q_{0}$ is orthogonal to $O x y$. Thus $\gamma$ passes through $q_{0}$ 
in a definite direction and therefore $\gamma$ does not depend on $q_{-1}$. This means that $\gamma$ coincides with our planar curve and therefore cannot be a geodesic.

Acknowledgements. The first author expresses his thanks to the colleagues of the Courant Institute of Mathematical Sciences where this revision was completed. Special thanks are due to P. Deift and $\mathrm{A}$. Weinstein for interesting discussions and helpful comments.

\section{References}

1. Veselov, A.P.: Integration systems with discrete time and difference operators. Funct. Anal. Appl. 22, 1-13 (1988) (Russian)

2. Faddeev, L.D., Takhtadjan, L.A.: The quantum inverse scattering method and XYZ Heisenberg model. Uspekhi Mat. Nauk. 34, 13-63 (1979) (Russian)

3. Baxter, R.: Eight-vertex model in lattice statistics and one-dimensional anisotropic Heisenberg chain. Ann. Phys. 76, 1-71 (1973)

4. Pokrovsky, V.L., Khokhlachev, S.B.: Nonhomogeneous stationary states in Heisenberg model. Pisma v JETP 22, 371-373 (1975) (Russian)

5. Granovsky, Ya.I., Zhedanov, A.S.: Periodic structures on quantum spin chain. JETP 89, 2156-2163 (1985) (Russian)

6. Granovsky, Ya.I., Zhedanov, A.S.: The solution of domain type in a magnetic chain. Theor. Math. Phys. 71, 145-153 (1987) (Russian)

7. Veselov, A.P.: The integration of the stationary problem for classical spin chains. Theor. Math. Phys. 71, 154-159 (1987) (Russian)

8. Moser, J.: Various aspects of integrable hamiltonian systems. In: Proc. CIME Conf., Bressanone, Italy, June 1978, Prog. Math., Vol. 8. Basel: Birkhäuser 1980

9. Moser, J.: Integrable hamiltonian systems and spectral theory. Pisa: Lezioni Fermiane 1981

10. Moser, J.: Geometry of quadric and spectral theory. Chern Symposium 1979, Berkeley, pp. 147-188. Berlin, Heidelberg, New York: Springer 1980

11. Symes, W.: The $Q R$ algorithm and scattering for the finite nonperiodic Toda lattice. Physica 4D, 275-280 (1982)

12. Deift, P., Li, L.C., Tomei, C.: Matrix factorizations and integrable systems. Commun. Pure Appl. Math. 42, 443-521 (1989)

13. Gohberg, I., Lancaster, P., Rodman, L.: Matrix polynomials. New York: Academic Press 1982

14. Dubrovin, B.A., Matveev, V.B., Novikov, S.P.: Nonlinear equations of $K d V$ type, finite-zone linear operators and abelian varieties. Russ. Math. Surv. 31, (1), 59-146 (1976)

15. Dubrovin, B.A.: Completely integrable hamiltonian system associated with matrix operators and abelian varieties. Funct. Anal. Appl. 11, 28-41 (1977)

16. Knörrer, H.: Geodesic on quadrics and a mechanical problem of Neumann. J. Reine Angew. Math. 334, 69-78 (1982)

17. Novikov, S.P.: Periodic problem for $K d V$ equation, I. Funct. Anal. Appl. 8(3), 54-66 (1974)

18. Adler, M., van Moerbeke, P.: Completely integrable systems, Euclidean Lie algebras and curves. Adv. Math. 38, 267-317 (1980)

19. Reiman, A., Semenov-Tyan-Shansky, M.: Reduction of hamiltonian systems, affine Lie algebras and Lax equations. Invent. Math. 54, 81-101 (1979)

20. Arnold, V.I.: Mathematical methods of classical mechanics. Berlin, Heidelberg, New York: Springer 1978

21. Pantazis, S.: Prym varieties and the geodesic flow on SO(n). Math. Ann. 273, 297-316 (1986)

22. Manakov, S.V.: Remarks on the integration of the Euler equations of $n$-dimensional rigid body. Funct. Anal. Appl. 10 (4), 93-94 (1976) (Russian)

23. Dubrovin, B.A.: Theory of operators and real algebraic geometry. Lecture Notes in Math., Vol. 1334, pp. 42-59. Berlin, Heidelberg, New York: Springer 1988

24. Bobenko, A.I.: Real algebraic-geometry solutions of Landau-Lifschitz equation in terms of Prym theta-functions. Funct. Anal. Appl. 19, 6-19 (1985)

25. Jacobi, C.G.J.: Sur la rotation d'un corps. In: Gesammelte Werke, 2. Band (1881) 291-352 
26. Adams, M.R., Harnad, J., Previato, E.: Isospectral hamiltonian flows in finite and infinite dimensions. Commun. Math. Phys. 117, 451-500 (1988)

27. Neumann, C.: De problemato quodam mechanico quod ad primam integralium ultraellipticorum classem revocatur. J. Reine Angew. Math. 56, 46-63 (1859)

28. Veselov, A.P.: Geometry of hamiltonian systems, connected with nonlinear partial differential equations. Ph. D. Thesis, Moscow State University 1981

29. Veselov, A.P.: Confocal surfaces and integrable billiards on the sphere and in the Lobachevsky space. Reprint of the Forschungsinstitut für Mathematik, ETH Zürich 1989

30. Libermann, P., Marle, C.M.: Symplectic geometry and analytic mechanics. Dordrecht, Holland: Reidel 1987 (see Chap. IV, Theorem 4.8)

31. Abrahams, R., Marsden, J.E.: Foundations of mechanics. New York: Benjamin Cummings 1978 (in particular, pp. 302-303)

32. Deift, P.A., Li, L.C., Tomei, C.: Loop groups, discrete versions of some classical integrable systems, and rank 2 extensions. Preprint, Courant Inst. Math. Sci., November 1990

33. Mishchenko, A.S., Fomenko, A.T.: Euler equations on finite dimensional Lie groups. Izvestiya Akad. Nauk SSR, Ser. Math. 42, 396-415 (1978)

Communicated by J. N. Mather

Note added in proof. Recently $P$. Deift succeeded in answering the question whether the discrete version of the spinning top [formula (4) of Sect. 1] is identical with the "time $\tau$ " map, for some $\tau>0$, of the continuous flow given by Eq. (5). Even though both systems have the same integrals the answer to this equation is negative (as one would hope). Using the formalism of [32] Deift constructed an interpolating flow described by the equation

$$
\dot{M}=[M, B], \quad B=B(M)
$$

for which the "time 1" map gives rise to the discrete system (4). Comparing $(*)$ and (5) he found that for small $|M|$ the system (*) has the form

$$
\dot{M}=[M, B]=[M, \Omega]+K(M)+O\left(|M|^{5}\right),
$$

where $K(M)$ is a quartic polynomial which is not identically equal to zero. He shows thus that both flows do not agree; but the discrete system (4) can be viewed as a discretization of (5) agreeing with the continuous flow up to third order. 
